\title{
Discussion On and Development Trend of Graphic and Image Processing Technologies of Computer
}

\author{
Hongliang Xing \\ College of Design \& Art of Shenyang Aerospace University
}

\begin{abstract}
The rapid development of graphic and image processing technologies of computer not only bring about convenience to people's daily life and work, being widely applied in various fields, but also bring along the development of relative industries, greatly attracting people's attention. This thesis discusses the relative contents as well as development trend of graphic and image processing technologies of computer, hoping that people can know more about such technologies while the technologies can have broaden development space.
\end{abstract}

Keywords: Computer; Graphic and image; Processing technology.

\section{Introduction}

The graphic and image processing technologies of computer refer to design assistant technology of picture processing by computer software, which enable graphic to become more effective, fast, accurate and standard, and image to become more vivid and specified. With the development of computer technology, the graphic and image processing technologies of computer become more mature. Currently, such technologies already have held important positions both in tools and technological method in graphic design field that their intrinsic strong and convenient functions greatly spill into peoples life as well as diversified industries, bringing about many expedience to people's life and work.

\section{The content and characteristics of graphic and image processing technologies of computer}

\subsection{Content}

The graphic and image processing technologies of computer mean employing geometric model and data to optimize, modify, store, compress and show etc graphic and image to promote image effect, strengthen image quality and enhance visual impact. The course of graphic and image processing technologies of computer is realized through the process toward graphic and image by the two- dimensional and three- dimensional technologies of graphic compilation according to the corresponding operation method. The process technologies mainly include following contents: the computer transfers the to be modified picture into data model or conducts recovery of the picture so as to make the processing of computer toward picture more precision; the technologies employ software to conduct translation, rotation, perspective, projection or enhancement toward the original photo and construct physical model based on corresponding proportion; in addition, the technologies can use relative data to compress picture and encode the picture to simplify the picture information; moreover, the technologies can adopts software to adjust and change color, curve, hook face and line etc to produce practical and three- dimensional picture; etc. After such processing, the geometric attribute of object is manifested in the form data image, and the the processing of graphic and image is accomplished through further perfection.

\subsection{Characteristics}

With the rapid development of information technology, the graphic and image processing technologies of computer have formed technological method with special characteristics, mainly including: 1. Strong reappear function. The traditional graphic and image processing technologies of computer often influence the image quality by copy, storage and other operations. However, for the modern graphic and image processing technologies of computer, the image quality is free of the influence of copy and storage. 2. Wider occupied frequency range. It can be found through comparing the graphic and image information with voice message that the graphic and image information has 
larger frequency range, which may cause some difficulties in processing graphic and image by computer. 3. Wider application range. The data resources can be acquired by various channels. Such as the graphic gained by microscope or the image acquired by astronomical mirror, all can be operated and processed by the graphic and image processing technologies of computer. 4. More flexible. The contents about logic change and mathematical formula of daily life can be operated and processed by the graphic and image processing technology of computer.

\section{The difference and connection of the graphic and image processing technologies of computer}

\section{1 difference}

There are some difference between graphic process technology of computer and image processing technology of computer, mainly including: 1. Different theoretical basis. The graphic is based on spline geometry, computation geometry, perspective transformation, and fractal theory etc; while the basics of image are probability statistics, fuzzy mathematics, digital signal processing and other relevant theories. 2. Different data structure source. The graphic and image processing technologies are formed aiming at the specified data structure of a certain picture that all pixels of the picture are the important elements of the data group. The difference interior software structures of the computer have different ways in processing information. The graphic is real while the image is controlled by the subjective consciousness of people. 3. Difference processing method. The processing methods of graphic are clip, geometric transformation, light and shade processing, matching of curve and hook face, elimination and concealing etc; while the geometric modification, change, identification, enhancement, segmentation and comprehension etc compose the processing method of image. 4. Different terminal application way. The graphic processing mainly is used in computer animation, art, simulation and other aspects; while the application fields of image processing mainly include remote sensing, medicine, industry, space flight and aviation, military as well as other fields.

\subsection{Connection}

The relation between graphic processing technology of computer and image processing technology of computer is close that the two technologies are overlapped, interpenetrative, and syntrophic. For practical operation process, the effective combination application of the two technologies can greatly promote the visual effect and quality of the picture rather than a single technology of graphic processing or image processing. With the continua promotion and completion of the graphic and image processing technologies of the computer, the two technologies will have more similarities, more blurry bound and more close combination. The graphic and image processing technologies of the computer will be combined into an entirety truly and effectively, playing more and more important roles in the diversified relative fields and forming a wholly- new computer subject which is full of energy.

\section{The daily application of the graphic and image processing technologies}

Since the graphic and image processing technologies of the computer rapidly develops and becomes mature, currently such technologies already have been widely applied in diversified fields, namely business, art, industry and design etc. The technologies not only closely connect to people's daily life, but also facilitate people in broadening mind and changing live environment. For example, the application of the graphic and image processing technologies of the computer in packaging design. The technologies not only enable people to conduct works such as type setting, reduction printing, and hue adjustment etc, but also help people to add wordart, making the advertising naturally and smoothly, thus improving the promotion effect and attracting people; the application in medicine. The technologies can transfer the video information of medicine into three- dimensional view, largely improving the diagnosis accuracy of the doctor and ensuring the health of the patient; the application in webpage design. The technologies can make the webpage interface be full of texture, strengthening the expressed emotion and content of the website to the largest extent and attracting netizen; the 
application in film and TV industry. The technologies can be used in producing various close- up scene, like the collapse of large- scale building, and the breathtaking trick effect etc, transferring picture into animation to make dynamic picture more real and make animation show true rules which can be accepted by people more easily. What's more, the graphic and image processing technologies of the computer have also been employed in constructional engineering design, land survey, appearance design of automobile and other communication media, environmental protection, bioanalysis, military as well as other fields with significant influence.

\section{The development trend of the graphic and image processing technologies}

With the graphic and image processing technologies of the computer becoming more mature, and the improvement requirements of different fields toward graphic and image, the development path of graphic and image processing software speeds up while its application range becomes more and more widely. In the future development process, the graphic and image processing technologies will pay more attention to the multiple- prospective, multiple- purpose, multiple- channel and multipledirection processing of integral information so as to realize high definition and high resolution, threedimensional imaging, as well as full automation. Meanwhile, the development of the graphic and image processing technologies will focus on the visual scientific computing technology so as to accomplish the remote controlling and development with high precision in medicine, weather analysis, hydromechanics and other fields, as well as focus on the practical operation to fully analyze the characteristics, key element of graphic and image so as to develop to the integral function of graphic processing. What's more, the technologies will lay emphasis on the advanced theoretical guidance, continually explore new technology to provide high- effective research in a wholly- new prospective. In conclusion, the application range of the graphic and image processing technologies of the computer will be more and broader while their future perspective will become more and brighter.

\section{Conclusion}

The rapid development and wide application of the graphic and image processing technologies of the computer enrich people's daily life, promote the technological innovation of various fields, and realize the improvement of creation capacity to a large extent. However, in nowadays, where possesses global economy and rapid technological development, the new challenges and opportunities require more toward the graphic and image processing technologies. Thus, only by speeding up the development and expansion of the graphic and image processing technologies of the computer, applying the new concept and originality to the the graphic and image processing field of the computer as well as continually attempting and exploring special graphic and image processing technologies can they meet the complex and changeable requirement of the market, adjust to the development trend of the graphic and image processing technologies as well as ushering in the new world of the graphic and image processing technologies .

\section{References}

[1]. Chen Mintui, Jin Xudong, Brief Talk on the Graphic and Image Processing Technologies of the Computer $[\mathrm{J}]$, academic journal of Changchun University of Science and Technology, 2011(01):138-139

[2]. He Xiaojuan, Discussion on the Graphic and Image Processing Technologies of the Computer [J], China Computer \& Communication, 2013(11):164-165

[3]. Chen Liping, Innovative Strategy Exploration and Analysis of the Graphic and Image Processing Technologies of the Computer [J], Digital Technology and Application, 2014(10) 
[4]. Wang Yingrong, Wang Jingyi, The Graphic and Image Processing Technologies of the Computer [J], Tianjin University Of Technology, 2012(03):6-10

[5]. Zhang Yanli, Exploration on the Graphic and Image Processing Technologies of the Computer [J], Heilongjiang Science, 2012(03):6-10 